\title{
Optic nerve diameters and perimetric thresholds in idiopathic intracranial hypertension
}

Tommaso Salgarello, Ciro Tamburrelli, Benedetto Falsini, Andrea Giudiceandrea, Alberto Colotto

\begin{abstract}
Aims/background-Idiopathic intracranial hypertension (IIH) is a central nervous disorder characterised by abnormally increased cerebrospinal fluid (CSF) pressure leading to optic nerve compression. An indirect estimate of increased CSF pressure can be obtained by the ultrasonographic determination of optic nerve sheaths diameters. Computerised static perimetry is regarded as the method of choice for monitoring the course of the optic neuropathy in IIH. The aims were to compare the echographic optic nerve diameters (ONDs) and the perimetric thresholds of patients with IIH with those of age-matched controls, and to examine the correlation between these two variables in individual patients with papilloedema.
\end{abstract}

Methods-Standardised A-scan echography of the mid orbital optic nerve transverse diameters and automated threshold perimetry (Humphrey 30-2) were performed in 20 patients with IIH with variable degree of papilloedema (according to the Frisén scheme) and no concomitant ocular diseases. Echographic and perimetric results were compared with those obtained from 20 age-matched controls.

Results-When compared with controls, patients with IIH showed a significant increase in mean ONDs and significantly reduced mean perimetric sensitivities. In individual patients with papilloedema, the transverse ONDs correlated negatively with Humphrey mean deviation values and positively with pattern standard deviation values.

Conclusion-These results indicate that OND changes in IIH are associated with perimetric threshold losses, and suggest that IIH functional deficits may be related to the degree of distension of optic nerve sheaths as a result of an increased CSF pressure.

(Br F Ophthalmol 1996;80:509-514)

B Falsini

A Giudiceandrea

A Collotto

Correspondence to: Dr Tommaso Salgarello, Institute of Ophthalmology, Catholic University, Largo F Vito, 1, I-00168 Rome, Italy.
Idiopathic intracranial hypertension (IIH), also known as pseudotumour cerebri and benign intracranial hypertension, is a disorder of elevated intracranial pressure of unknown cause. Since the only permanent morbidity from IIH is loss of vision, the current knowledge of the disease indicates that its course must be carefully followed by investigating primarily the presence and eventual progression of visual loss. ${ }^{1}$

Many series analysed the potential relation between visual loss and various clinical variables in IIH, in order to gather information about pathophysiology of the disease and the correct management of patients. In particular, the relations between functional measurements (Snellen visual acuity, ${ }^{2-5}$ contrast sensitivity, ${ }^{5-8}$ pupil examination, ${ }^{59}$ pattern electroretinogram and visual evoked potentials, ${ }^{70}$ perimetric thresholds ${ }^{1511-19}$ ) and morphological variables of the optic nerve (papilloedema degree, ${ }^{13} 151620$ echographic assessment of optic nerve transverse diameters ${ }^{21-24}$ ) have been investigated.

Previous evidence has emphasised the sensitivity and specificity of automated static threshold perimetry for detecting the earliest functional defects and following their progression, and the role of optic nerve echography in predicting the pressure of cerebrospinal fluid (CSF) and its dynamic variables. In the present study, echographic optic nerve diameters (ONDs) and static perimetric thresholds of patients with IIH were compared with those of age-matched controls. The correlation between OND and perimetric threshold changes found in individual patients with papilloedema was also evaluated.

\section{Materials and methods}

Twenty patients (two males, 18 females; mean age 47 (SD 11.9) years; range 27-71 years) with a confirmed diagnosis of idiopathic intracranial hypertension were investigated. All patients met the modified Dandy criteria for IIH diagnosis - that is, (1) signs and symptoms of increased intracranial pressure in an alert and awake patient, (2) no localising findings on neurological examination other than abducens nerve paresis, (3) no evidence of deformity, displacement, or obstruction of the ventricular system and otherwise normal neurodiagnostic studies, except for increased CSF pressure ( $>250 \mathrm{~mm} \mathrm{H}_{2} \mathrm{O}$ ), and (4) no other cause of increased intracranial pressure present. ${ }^{25}$ All the patients had a lumbar puncture documenting elevated CSF pressure (range $260-320 \mathrm{~mm}$ $\mathrm{H}_{2} \mathrm{O}$ ) 1 or 2 weeks before the ophthalmic examination. From each patient a medical history was taken (including the results of previous neurological and neurosurgical examination), and a complete ophthalmic evaluation was performed, including assess- 
Table 1 Clinical data of patients

\begin{tabular}{|c|c|c|c|c|c|c|c|c|c|c|c|c|c|c|c|}
\hline \multirow{2}{*}{$\begin{array}{l}\text { Patient lage } \\
\text { (years) /sex }\end{array}$} & \multicolumn{2}{|l|}{ Symptoms } & \multirow{2}{*}{$\begin{array}{l}\text { Duration of } \\
\text { symptoms } \\
\text { (years) }\end{array}$} & \multicolumn{2}{|c|}{$\begin{array}{l}\text { Optic disc } \\
\text { grade }\end{array}$} & \multicolumn{2}{|c|}{$\begin{array}{l}\text { Optic nerve } \\
\text { diameter }\end{array}$} & \multicolumn{2}{|l|}{$M D$} & \multicolumn{2}{|l|}{$P S D$} & \multicolumn{2}{|c|}{$V F G$} & \multicolumn{2}{|c|}{$\begin{array}{l}\text { Perimetric } \\
\text { defect }\end{array}$} \\
\hline & Ocular & Neurological & & $R$ & $L$ & $R$ & $L$ & $R$ & $L$ & $R$ & $L$ & $R$ & $L$ & $R$ & $L$ \\
\hline $1 / 54 / F$ & & Headache & 15 & 1 & 1 & 4.07 & 3.95 & 0.78 & 1.51 & 1.89 & 2.25 & 0.0 & 0.0 & & \\
\hline $2 / 59 / \mathrm{F}$ & & & ND & 4 & 3 & 6.14 & 5.83 & -7.33 & -17.9 & 9.63 & 11.3 & 3.2 & 3.3 & $\mathbf{N}$ & CR \\
\hline $3 / 47 / F$ & TVO & Headache & 32 & 3 & 4 & 4.09 & 4.70 & -2.21 & -2.86 & 2.69 & 2.54 & 2.1 & 2.1 & SN & $\mathbf{N}$ \\
\hline $4 / 53 / F$ & & & ND & 0 & 0 & 4.20 & 4.55 & -0.84 & -0.66 & 1.89 & 1.85 & 0.0 & 0.0 & & \\
\hline $5 / 37 / F$ & TVO & Vertigo & 5 & 2 & 1 & 5.30 & 4.60 & -1.28 & -2.27 & 2.13 & 3.61 & 0.0 & 2.1 & & IN \\
\hline 6/40/F & & Headache & 20 & 2 & 1 & 6.40 & 5.64 & -20.5 & -10.4 & 8.32 & 6.03 & 5.1 & 5.1 & CR & CR \\
\hline $7 / 43 / \mathrm{F}$ & & Headache & 19 & 2 & 1 & 3.84 & 3.85 & -3.54 & -2.49 & 3.78 & 3.29 & 2.1 & 2.1 & IN & $\mathbf{N}$ \\
\hline $8 / 38 / F$ & TVO & Headache & 8 & 0 & 0 & 3.90 & 4.20 & -2.92 & -5.65 & 4.07 & 6.18 & 2.1 & 3.2 & $\mathbf{N}$ & $\mathbf{N}$ \\
\hline 9/29/F & Diplopia & Headache & 22 & 2 & 1 & 4.50 & 4.68 & 0.88 & -0.62 & 1.64 & 2.81 & 0.0 & 0.0 & & \\
\hline $10 / 38 / M$ & & & ND & 1 & 3 & 5.60 & 5.90 & -3.22 & -4.56 & 2.45 & 2.30 & 0.0 & 3.2 & & $\mathbf{N}$ \\
\hline $11 / 68 / \mathrm{F}$ & TVO & Headache & 9 & 0 & 0 & 4.00 & 4.00 & -0.98 & -0.17 & 2.25 & 2.55 & 0.0 & 0.0 & & \\
\hline $12 / 46 / \mathrm{F}$ & TVO & Headache & 10 & 3 & 0 & 4.92 & 5.32 & -2.22 & -5.75 & 4.66 & 9.95 & 2.1 & 3.2 & IN & IN \\
\hline $13 / 27 / F$ & TVO & & 3 & 2 & 4 & 6.20 & 6.60 & -3.54 & -10.0 & 2.08 & 11.5 & 2.1 & 3.2 & L & $\mathbf{N}$ \\
\hline $14 / 36 / \mathrm{M}$ & & Headache & 3 & 0 & 0 & 4.00 & 5.02 & -2.53 & -0.96 & 1.97 & 1.69 & 2.1 & 0.0 & $\mathbf{N}$ & \\
\hline $15 / 71 / \mathrm{F}$ & $\begin{array}{l}\text { TVO; } \\
\text { photopsias }\end{array}$ & & 6 & 0 & 0 & 4.43 & 4.25 & -8.32 & -6.45 & 4.17 & 3.77 & 4.1 & 3.2 & CR & L \\
\hline $16 / 48 / F$ & & $\begin{array}{l}\text { Headache, } \\
\text { vertigo }\end{array}$ & 2 & 1 & 1 & 4.25 & 4.56 & -1.62 & -0.14 & 2.92 & 2.23 & 1.1 & 1.1 & SN & ST \\
\hline $17 / 57 / F$ & TVO & $\begin{array}{l}\text { Headache, } \\
\text { vertigo }\end{array}$ & 40 & 0 & 0 & 3.70 & 3.77 & -3.47 & -1.92 & 4.45 & 2.96 & 3.2 & 2.1 & $\mathbf{N}$ & ST \\
\hline $18 / 60 / F$ & $\begin{array}{l}\text { TVO; } \\
\text { visual loss }\end{array}$ & $\begin{array}{l}\text { Headache, } \\
\text { vertigo }\end{array}$ & 22 & 0 & 0 & 4.36 & 4.20 & -5.66 & -12.7 & 3.69 & 6.64 & 3.2 & 4.1 & L & CR \\
\hline $19 / 50 / \mathrm{F}$ & TVO & Headache & 10 & 0 & 0 & 4.34 & 4.37 & -3.32 & -0.88 & 3.03 & 1.91 & 2.1 & 0.0 & IN & \\
\hline $20 / 42 / F$ & TVO & $\begin{array}{l}\text { Headache, } \\
\text { vertigo }\end{array}$ & 14 & 0 & 0 & 4.37 & 4.65 & -2.99 & -4.09 & 2.90 & 4.12 & 2.1 & 3.2 & $\mathrm{~L}$ & $\mathbf{N}$ \\
\hline
\end{tabular}

$\mathrm{ND}=$ not determinable; TVO=transient visual obscurations; optic disc grade according to the papilloedema staging scheme proposed by Frisén ${ }^{26}$; $\mathrm{MD}=$ mean deviation; $\mathrm{PSD}=$ pattern standard deviation; VFG=visual field grade (assessed by automated perimetry) according to the classification proposed by Wall and George ${ }^{5}$; perimetric defects: $C R=$ concentric restriction; $N=$ nasal loss; $I=$ inferonasal loss; $S N=$ superonasal loss; $S T=$ superotemporal loss; $L=$ lower altitude loss.

ment of refractive status, best corrected visual acuity determination, slit-lamp examination, applanation tonometry, ophthalmoscopy, standardised A-scan echography of the optic nerve, and automated static perimetry.

All the patients showed radiological evidence of an empty sella, a non-specific finding often associated with the disease, either at $x$ ray of the skull in the region of the sella turcica or on computed tomography (CT) and magnetic resonance imaging (MRI) scans. Whereas skull $x$ rays often demonstrated an enlarged sella, CT scans of the head revealed an area isodense to CSF almost completely filling an enlarged sella, with the pituitary gland flattened against the sellar floor or walls. MRI scans confirmed the diagnosis of primary empty sella syndrome. Concomitant systemic alterations, such as obesity, recent weight gain, and endocrine dysfunction were present in some cases. None of the patients was under treatment at the time of examination. The most commonly reported symptoms were headache with or without associated vomiting or nausea (14 cases, $70 \%$ ), transient visual obscurations ( 11 cases, $55 \%$ ), vertigo (five cases, $25 \%$ ), visual loss (one case, $5 \%$ ), photopsias (one case, $5 \%$ ), and diplopia (one case, $5 \%$ ). All but two eyes had normal corrected Snellen visual acuity $(20 / 20$ or better). Intraocular pressures ranged from 12 to $20 \mathrm{~mm} \mathrm{Hg}$. Direct ophthalmoscopic and biomicroscopic assessment of the degree of papilloedema, based on the staging scheme proposed by Frisén, (stage 0: normal optic disc, to stage 5: marked disc swelling with anterior expansion of the nerve head), ${ }^{26}$ showed bilateral papilloedema (ranging from stage 1 to 4 ) in 10 patients; one patient had papilloedema in only one eye (stage 3 ), and nine patients showed no signs of optic disc oedema in either eye. No concomitant ocular diseases were clinically evident.

Echographic measurements of transverse OND were performed on the same day as the automated testing, using a Mini A standardised echographic unit (Biophysics Medical), using a technique already described in the literature. ${ }^{21}$ Clinical accuracy in repeated measurements of optic nerve in the mid orbit is $0.03-0.05 \mathrm{~mm}$; measurement accuracy provided by the equipment is $0.01 \mathrm{~mm}$. Briefly, the probe was placed on the temporal ocular surface, and different optic nerve scans behind the globe were obtained. In the present study optic nerve diameters were measured midway between the nerve's insertion at the bulb and its passage through the orbital apex (intermediate optic nerve section). With the eye in the primary position, the echographic A-scan display of a maximally wide defect representing the lower reflective optic nerve as well as steeply rising or falling surface spikes (corresponding to the lateral and nasal arachnoid surfaces of the optic nerve sheaths) indicates the maximum diameter of the optic nerve in the area scanned by the beam. At increased intracranial pressure, as in patients with IIH, these subarachnoidal surfaces can be visualised even more clearly because of the volume of liquid separating them from the underlying nerve tissue.

Computerised threshold perimetry was performed on the Humphrey field analyser model 630 (Allergan-Humphrey, San Leandro, CA, USA) using the 30-2 program, examining a central visual field of 30 degrees ( 76 points arranged in a 6 degree rectangular grid offset by three degrees from the horizontal and vertical meridians) with STATPAC evaluation. The Humphrey STATPAC program provides measures of field reliability as well as two global 
indices of field sensitivity (mean deviation (MD) and pattern standard deviation (PSD)), and significance levels $(\mathrm{p}<10,5,2,1$, and $0.5 \%$ ) for each index indicating the percentage of normal age-matched eyes that would be expected to have an MD or PSD as extreme as that found in the tested eye.

Patients were experienced in automated static threshold perimetry. Automated visual fields were both qualitatively and quantitatively analysed at their second examination and their defects were graded according to the criteria proposed by Wall and George (visual field grade 0: normal field, to grade 5: blinding field loss). ${ }^{5}$ Patients' perimetric reliability indices (fixation losses, false positive and false negative errors) did not exceed the cut off values adopted in the Humphrey visual field analyser. Short term fluctuation (SF), which is an estimate of the intratest standard deviation of the threshold measurements, remained inside the expected normal range ( $p$ value $>5 \%$ ) in each patient.

Perimetric defects were evident in $70 \%$ of eyes (28 of 40) and, as in previous series, loss on the nasal side and generalised constriction of the visual field were the most frequently encountered changes. The following qualitative defects were found: nasal loss (10 eyes, $25 \%$ ), concentric restriction (five eyes, $12.5 \%$ ), inferonasal loss (five eyes, $12.5 \%$ ), associated blind spot enlargement (four eyes, $10 \%$ ), lower altitudinal loss (four eyes, $10 \%$ ), superonasal loss (two eyes, $5 \%$ ), and superotemporal loss (two eyes, $5 \%$ ). The assessment of the visual field grade (VFG) showed visual field loss evaluated at minimal grade in two eyes $(5 \%)$, mild grade in 12 eyes (30\%), moderate grade in 10 eyes $(25 \%)$, marked grade in two eyes $(5 \%)$, and maximal grade in two eyes $(5 \%)$ Clinical data of individual patients are summarised in Table 1. Twenty age-matched control subjects (two males, 18 females; mean age 47 years; range $27-71$ years) with normal general and neuro-ophthalmic examination were also tested.

Results obtained from normal subjects and patients were statistically evaluated by $t$ test for independent samples, Pearson's correlation, and linear regression analysis. In all tests, the measures derived from right and left eyes of patients were evaluated separately. For normal subjects only the measures derived from right eyes were included in the analysis. The correlation coefficient ( $r$ value) was used to estimate the degree of linear relation.

\section{Results}

The patients had a mean OND value significantly increased in comparison with controls ( $p<0.01$; mean right eyes 4.63 (SD 0.84) $\mathrm{mm}$; mean left eyes $4.73(0.77) \mathrm{mm}$; mean controls $3.7(0.6) \mathrm{mm})$. The echographic examination of OND showed an abnormal optic nerve in 19 of 40 eyes (47\%) evaluated according to the upper limit values for a normal OND, ${ }^{24}$ with a thickening of the optic nerve due to an increase of subarachnoidal fluid within the neural sheaths. Three out of

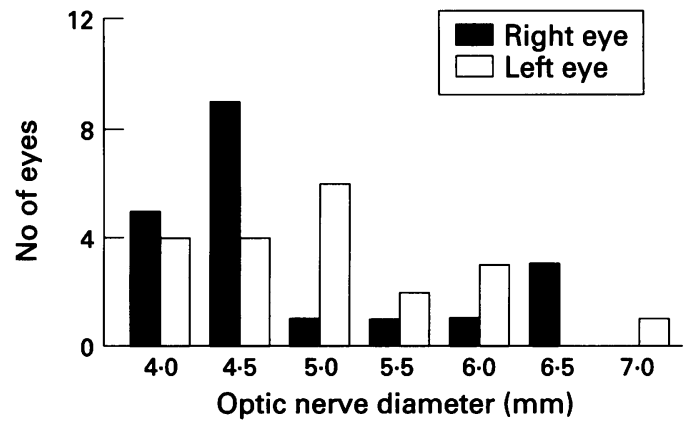

Figure 1 Frequency distributions of the echographically measured optic nerve diameters for idiopathic intracranial hypertension.

the 19 eyes with abnormal ONDs (15.8\%) had no evidence of optic disc oedema at clinical examination.

Figures 1-3 show the distribution histograms of echographic values and perimetric STATPAC values of ONDs, mean deviation (MD), and pattern standard deviation (PSD) for the right and left eyes of patients with IIH. In our sample, whereas the distribution of the MD and PSD values were similar between right and left eyes, the OND distribution showed a difference between right and left eyes in the range $4.0-4.5 \mathrm{~mm}$ (where the right eyes were prevalent) and 4.5-6.0 $\mathrm{mm}$ (where the left eyes were prevalent). However, the distributions did not differ significantly by $\chi^{2}$ test.

Mean perimetric indices of the patients were significantly increased in right and left eyes $(\mathrm{p}<0.01)$ compared with normal agecorrected values (mean (SD)): $\mathrm{MD}=-3.7$

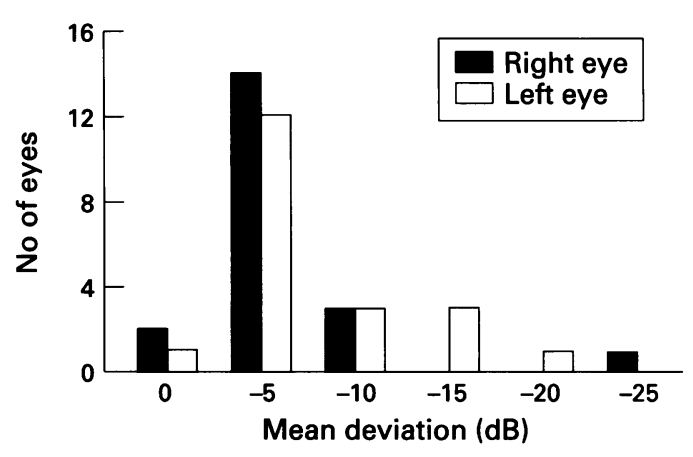

Figure 2 Frequency distributions of the measured mean deviation (Humphrey 30-2 threshold test) for idiopathic intracranial hypertension.

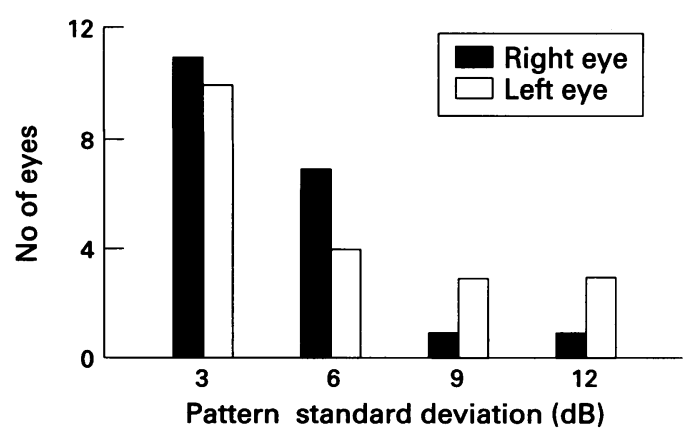

Figure 3 Frequency distributions of the measured pattern standard deviation (Humphrey 30-2 threshold test) for idiopathic intracranial hypertension. 


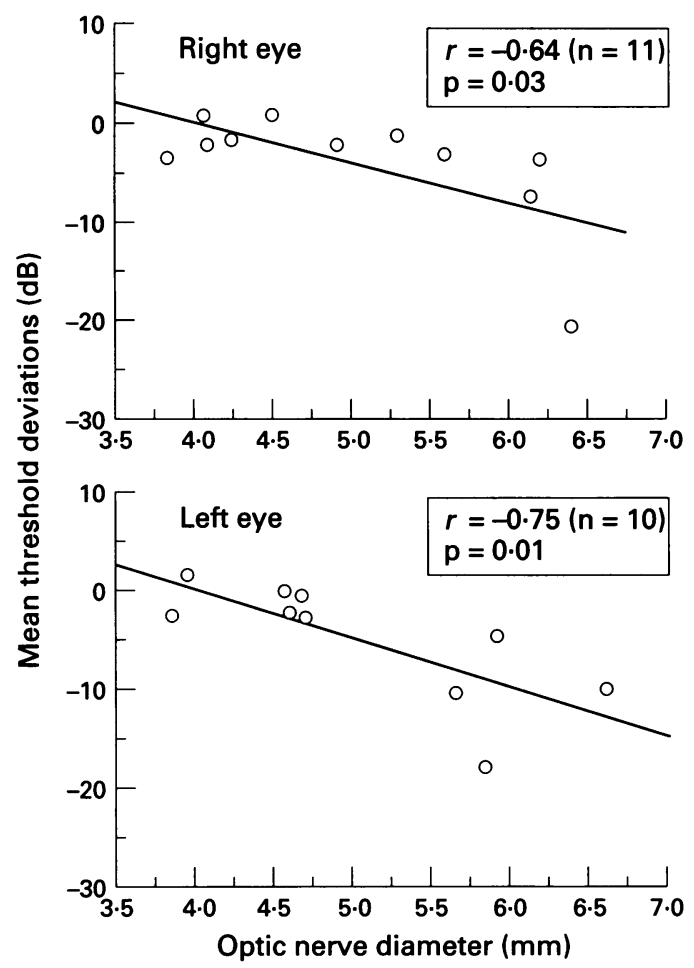

Figure 4 Linear regression of the Humphrey mean threshold deviations versus optic nerve diameter values of right and left eyes in 11 patients with IIH with papilloedema (stage 1-4 of Frisén scheme).

(4.6), $\mathrm{PSD}=3.5$ (2.1), corrected $\mathrm{PSD}=2.6$ (2.5) in right eyes, $\mathrm{MD}=-4.4(4.9), \mathrm{PSD}=4.5$ (3.10), corrected PSD $=3.6$ (3.4) in left eyes. Mean threshold deviation (MD) was abnormal in 21 of 40 eyes (52\%), whereas pattern standard deviation (PSD) was abnormal in 18 of 40 eyes $(45 \%)$.

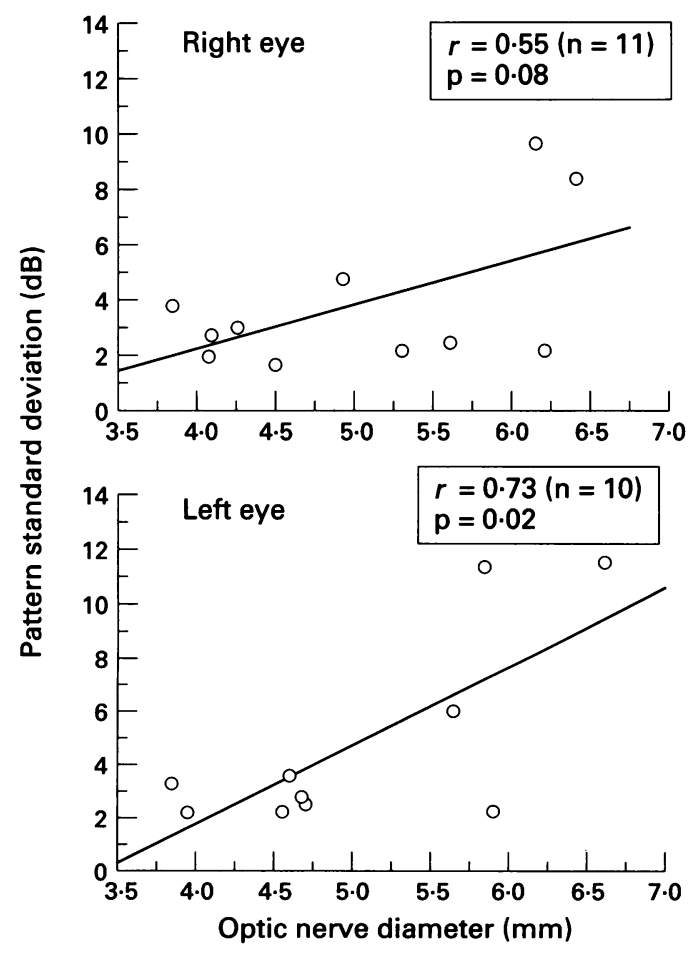

Figure 5 Linear regression of the Humphrey pattern standard deviations versus optic nerve diameter values of right and left eyes in 11 patients with IIH with papilloedema (stage 1-4 of Frisén scheme).
The correlation between perimetric indices and the corresponding OND values from the right and left eyes of patients with IIH was evaluated by including in the analysis only those eyes with clinical papilloedema (stage 1 to 4). This minimised the risk of overestimating the statistical significance of the correlation, which could have been artificially inflated by also including eyes with a normal disc. Figure 4 displays the individual $M D$ values plotted as a function of the corresponding OND values. The correlation was statistically significant for both eyes (right eyes $(n=11)$, $r=-0.64, \mathrm{p}=0.03$, left eyes $(\mathrm{n}=10), r=-0.75$, $\mathrm{p}=0.01$ ), indicating that $\mathrm{MD}$ tended to improve as OND decreased approaching normal values. Figure 5 depicts the individual PSD values plotted as a function of the corresponding OND values. The correlation between the two variables was statistically significant for the left eyes $(\mathrm{n}=10 ; r=0.73, \mathrm{p}=0.02)$, and approached statistical significance for the right ones with a comparable trend $(n=11 ; r=0.55$, $\mathrm{p}=0.08$ ). It can be noted from Figure 5 that PSD also tended to decrease as OND decreased towards, the normal range. Taken together, these correlations indicate that the MD and PSD changes in patients with IIH with papilloedema are at least in part associated with the corresponding OND variations. No correlation was found between OND values and visual field global indices ${ }^{5}$ in the affected eyes of patients.

\section{Discussion}

In the present study we have analysed functional variables and echographic measurements of OND in 20 patients with IIH with a documented increase in CSF pressure. Results showed that the mean OND and perimetric index values of patients were significantly altered compared with those of age-matched controls. In individual patients with papilloedema, OND values were significantly correlated with the corresponding perimetric threshold indices. Both Humphrey $30-2$ visual field global indices tended to improve as OND decreased approaching normal values.

Numerous studies have shown the existence of a direct correlation between ultrasound findings of an optic nerve thickening due to an increase of subarachnoidal fluid within the neural sheaths and increases in intracranial pressure. ${ }^{2122}$ On monitoring intracranial pressure by the intraventricular insertion of a catheter and a transducer in a group of patients with cerebral diseases, Cennamo et al demonstrated that the measurements of OND by A-scan standardised echography can provide an immediate evaluation, in real time, of endocranial pressure. ${ }^{23}$ Tamburrelli et al confirmed these results by means of Ringer's lactate infusion into the spinal and endocranial subarachnoid spaces of 20 patients: this method allowed investigators to evaluate within a very short period of time the repercussions on the optic nerve of changes in certain hydrodynamic variables of the CSF system, and successively to correlate these data with those of OND. They obtained a biphasic curve 
representing a direct positive relation between OND measurements along the intraorbital portion and diastolic intracranial pressure over $15 \mathrm{~mm} \mathrm{Hg}$, and based on normal intracranial pressure values, they could define an echographic biometric range of normal ONDs. ${ }^{24}$ Of the eyes included in the present study, 19 out of $40(47 \%)$ had significantly increased OND values. Interestingly, three out of these 19 eyes $(15.8 \%)$ had no papilloedema at clinical examination. Since our patients had increased CSF pressure as documented by a lumbar puncture, we may presume that in some cases of IIH the distension of the optic nerve sheaths, as a result of elevated CSF pressure and detectable by optic nerve echography, could precede the appearance of a clinically evident papilloedema. The present finding in some way stresses the value of standardised A-scan echography in the morphological examination of the optic nerve in IIH.

Various studies recommend the clinical evaluation of patients with IIH by automated perimetry. This technique allows the detection of functional changes, such as the development of initial perimetric defects or the eventual progression of the disease. In our sample, all patients presented with a primary empty sella syndrome and IIH with or without papilloedema. Visual loss in these patients may be explained by two mechanisms related to papilloedema and/or empty sella. As to papilloedema, several studies have reported evidence that the anterior optic nerve is the site of the lesions. Functional damage, indeed, is associated with a optic disc oedema due to the transmission of the increased intracranial pressure through the neural sheaths. Early visual loss in patients with IIH with papilloedema has been related to a nerve conduction defect secondary to the stasis of axoplasmic flow in retinal ganglion cells and/or a compression induced ischaemia of the same cells. ${ }^{27}$ As to those patients who do not have papilloedema, different mechanisms leading to visual loss have been suggested. Foley and Posner have described many patients with empty sella and increased intracranial pressure without papilloedema. ${ }^{28}$ They suggested that (1) papilloedema is less frequent in the combined syndrome than in IIH alone, and (2) visual field defects and visual loss are more likely when IIH and the primary empty sella syndrome appear together than when either occurs independently. Visual complaints were explained, indeed, by the presence of both chronic elevation of intracranial pressure and an incompetent diaphragma sellae which produces herniation of the subarachnoid cistern into the sella turcica, with the resultant stretching of the optic nerves or chiasm. According to this mechanism, the eventual absence of papilloedema may occur because pressure on the optic nerves occluded the subarachnoid pathways leading to the optic discs. Other hypotheses were postulated for the lack of papilloedema such as the probable presence of congenital or acquired optic nerve sheaths defects, 'chronic pseudotumour cerebri' with resolution of papilloedema, or intermittent elevation of intracranial pressure below the threshold to produce papilloedema. ${ }^{29}$ Wall and George used perimetry in order to follow the clinical evolution of IIH but mainly to decide the treatment. ${ }^{5}$ In fact they confirmed other recents observations that in the individual patients' symptoms and the degree of papilloedema (except for the high grade and/or atrophic papilloedema) may not correlate with loss of visual function. ${ }^{5}{ }^{20}$ On the other hand, automated perimetry of the central 30 degrees seems to be able to detect early functional defects in IIH, with high sensitivity and specificity. ${ }^{19}$

The findings obtained in the present study indicate the OND changes in IIH are associated, at least in part, with perimetric threshold alterations, and suggest that IIH functional deficits may be related to the degree of distension of optic nerve sheaths as the result of an increase in CSF pressure. In conclusion, transverse OND evaluation using echographic techniques may be useful not only for morphological analysis, but also to provide indications of the associated visual loss in IIH. This approach may be of potential clinical value for establishing the timing and methods of treatment. Indeed, treatment of IIH focuses on the early detection and prevention of visual loss.

1 Wall M, Hart WM, Burde RM. Visual field defects in idiopathic intracranial hypertension (pseudotumor cerebri).Am ₹ Ophthalmol 1983;96:654-69.

2 Guidetti B, Giuffrè R, Gambacorta D. Follow-up study of 100 cases of pseudotumor cerebri. Acta Neurochir 1968;18: 259-67.

3 Holmes G. The prognosis in papilloedema. Br $\mathcal{F}$ Ophthalmol 1937;21:337-42.

4 Huber A. In: Eye signs and symptoms in brain tumors. 3rd ed. St Louis: Mosby, 1976: 109-13.

5 Wall $\mathrm{M}$, George D. Idiopathic intracranial hypertension. A prospective study of 50 patients. Brain $1991 ; 114: 155-80$.

6 Bulens C, Meerwaldt JD, Koudstaal PJ, Van der Vildt GJ. Spatial contrast sensitivity in benign intracranial hypertension. $\mathcal{I}$ Neurol Neurosurg Psychiatry 1988;51:1323-9.

7 Verplanck M, Kaufman DI, Parsons T, Yedavally S, Korinakis D. Electrophysiology versus psychophysics in the detection of visual loss in pseudotumor cerebri. Neurology 1988;38: 1789-92.

8 Wall $M$. Contrast sensitivity testing in pseudotumor cerebri. Ophthalmology 1986;93:4-7.

9 Kardon RH, Haupert CL, Thompson HS. The relationship between static perimetry and the relative afferent pupillary defect. Am F Ophthalmol 1993;115:351-6.

10 Falsini B, Tamburrelli C, Porciatti V, Anile C, Porrello G Mangiola N. Pattern electroretinograms and visual evoked potentials in idiopathic intracranial hypertension. Ophthalmologica 1992:205:194-203.

11 Bulens C, De Vries WAEJ, Van Crevel H. Benign intracranial hypertension: a retrospective and follow-up study. $\mathcal{f}$ Neurol Sypertension: a retro $1979 ; 40: 147-57$.

12 Corbett JJ, Savino PJ, Thompson HS, Kansu T, Schatz NJ, Orr LS, et al. Visual loss in pseudotumor cerebri. Follow-up of 58 patients from five to 41 years and a profile of 14 patients with permanent severe visual loss. Arch Neurol 1982;39:461-74.

13 Orcutt JC, Page NGR, Sanders MD. Factors effecting visual loss in benign intracranial hypertension. Ophthalmology 1984;91:1303-12.

14 Round R, Keane JR. The minor symptoms of increased intracranial pressure: 101 patients with benign intracranial hypertension. Neurology 1988;38:1461-4.

15 Rush JA. Pseudotumor cerebri: clinical profile and visual outcome in 63 patients. Mayo Clin Proc 1980;55:541-6.

16 Smith TJ, Baker TS. Perimetric findings in pseudotumo cerebri using automated techniques. Ophthalmology 1986 93:887-94.

17 Sorensen PS, Krogsaa B, Gjerris F. Clinical course and prognosis of pseudotumor cerebri. A prospectic study of 24 patients. Acta Neurol Scand 1988;77:164-72.

18 Wall $M$, George D. Visual loss in pseudotumor cerebri: incidence and defects related to visual field strategies. Arch Neurol 1987;44:170-5.

19 Wall M, Conway MD, House PH, Allely R. Evaluation of sensitivity and specificity of spatial resolution and Humphrey automated perimetry in pseudotumor cerebri patients and normal subjects. Inv Ophthalmol Vis Sci 1991; 32:3306-12.

20 Corbett J, Nerad JA, Tse DT, Anderson RL. Results of optic nerve sheath fenestration for pseudotumor cerebri the lateral orbitotomy approach. Arch Ophthalmol 1988; 106:1391-7. 
21 Ossoinig KC, Cennamo G, Frazier Byrne S. Ecographic differential diagnosis of optic nerve lesions. Doc Ophthalmol Proc Ser 1981;29:327-31.

22 Ossoinig $\mathrm{KC}$, Tamburrelli C. Otticopatia compressiva: nella ipertensione endocranica benigna. In: Atti del congresso Giornate di Ecografia Oftalmica 3/4 ottobre, 1986: 62-3.

23 Cennamo G, Gangemi M, Stella L. The correlation between endocranial pressure and optic nerve diameter: an ultrasonographic study. Doc Ophthalmol Proc Ser 1987;48: 603-6.

24 Tamburrelli C, Anile C, Mangiola A, Falsini B, Palma P. CSF dynamic parameters and changes of optic nerve diameters measured by standardized echography. Doc Ophthalmol Proc Ser 1993;55:101-9.

25 Smith JL. Whence pseudotumor cerebri? $\mathcal{f}$ Clin NeuroOphthalmol 1985;5:55-6.

26 Frisén L. Swelling of the optic nerve head: a staging scheme. f Neurol Neurosurg Psychiatry 1982;45:13-8.

27 Hayreh SS. Optic disc edema in raised intracranial pressure: H. Pathogenesis. Arch Ophthalmol 1977;95:1553-65.

28 Foley KM, Posner JB. Does pseudotumor cerebri cause the empty sella syndrome? Neurology 1975;25:565-9.

29 Marcelis J, Silberstein SD. Idiopathic intracranial hypertension without papilledema. Arch Neurol 1991;48:392-9. 